\title{
Síndrome de Kounis, a propósito de un caso
}

\author{
Kounis syndrome, apropos of a case
}

\section{Correspondencia}

Cristhian Felipe Ramirez Ramos cfrr89@gmail.com

\section{Recibido: $15 / 11 / 2017$}

Aprobado: 07/12/2017

Citar como: Méndez-Betancourt JL, Ramirez-Ramos CF, López-Guevara OA, Osorio-Carmona HE. Síndrome de Kounis, a propósito de un caso. Acta Med Peru. 2018;35(1):65-70

\author{
Johan L. Méndez-Betancourt'1,2, Cristhian F. Ramirez-Ramos ${ }^{2,3}$, Oscar A. López- \\ Guevara ${ }^{4}$, Hugo E. Osorio-Carmona ${ }^{3,5}$ \\ 1 Unidad de Cuidados intensivos, Clínica Uros. Neiva, Colombia. \\ 2 Semillero de investigación en medicina interna, Universidad Surcolombiana. Neiva, Colombia. \\ 3 Hospital Universitario Hernando Moncaleano Perdomo. Neiva, Colombia. \\ 4 Servicio de medicina interna, Clínica Uros. Neiva, Colombia. \\ 5 Departamento de cardiología, Clínica Uros. Neiva, Colombia.
}

\section{RESUMEN}

El síndrome de Kounis se caracteriza por un compromiso cardiaco que es secundario a una respuesta alérgica. Hay pocos datos acerca de la epidemiologia de esta enfermedad debido a su reciente descripción. Se presenta el caso de una paciente mujer de 46 años sin antecedentes de importancia que presentó dolor tipo cólico episódico en hipocondrio derecho de 4 días de tiempo de enfermedad, en la que se consideró el diagnóstico de cólico de origen biliar. Quince minutos luego de manejo sintomático (hiocsina/dipirona), la paciente presentó dolor torácico en región precordial de inicio súbito. La evaluación física mostró hipotensión, taquicardia, presencia de habones en todo el cuerpo y sibilantes a la auscultación pulmonar. El electrocardiograma mostro infradesnivel del segmento ST. Considerado como síndrome coronario agudo, se realizó el manejo con esteroides y soporte vital, y se indicó monitorización cardiovascular en unidad de cuidados intensivos. La paciente egresó sin complicación luego de unos días.

Palabras clave: Vasoespasmo coronario; Reacción alérgica; Enfermedades cardiovasculares (fuente: DeCS BIREME).

\footnotetext{
ABSTRACT

Kounis syndrome is defined as an acute coronary syndrome secondary to an allergic response; and it is also known as allergic angina - acute myocardial infarction. There is little data on the epidemiology of this disease because of having been recently described. We present the case of a 46-year old woman who developed an episode of colicky abdominal pain in the right upper quadrant for 4 days, and a diagnosis of abdominal pain of biliary origin was considered. Fifteen minutes after symptomatic therapy was administered (hyoscine and metamizole), the patient developed sudden chest pain. Physical examination revealed hypotension, tachycardia, rash, and wheezing. The electrocardiogram showed ST segment depression. The case was managed as an acute coronary syndrome, vital support and steroids were part of its management, and the patient was admitted in the intensive care unit for coronary monitoring. A few days later, the patient was uneventfully discharged.

Keywords: Coronary vasospasm; Allergic reaction; Cardiovascular diseases (source: MeSH NLM).
} 


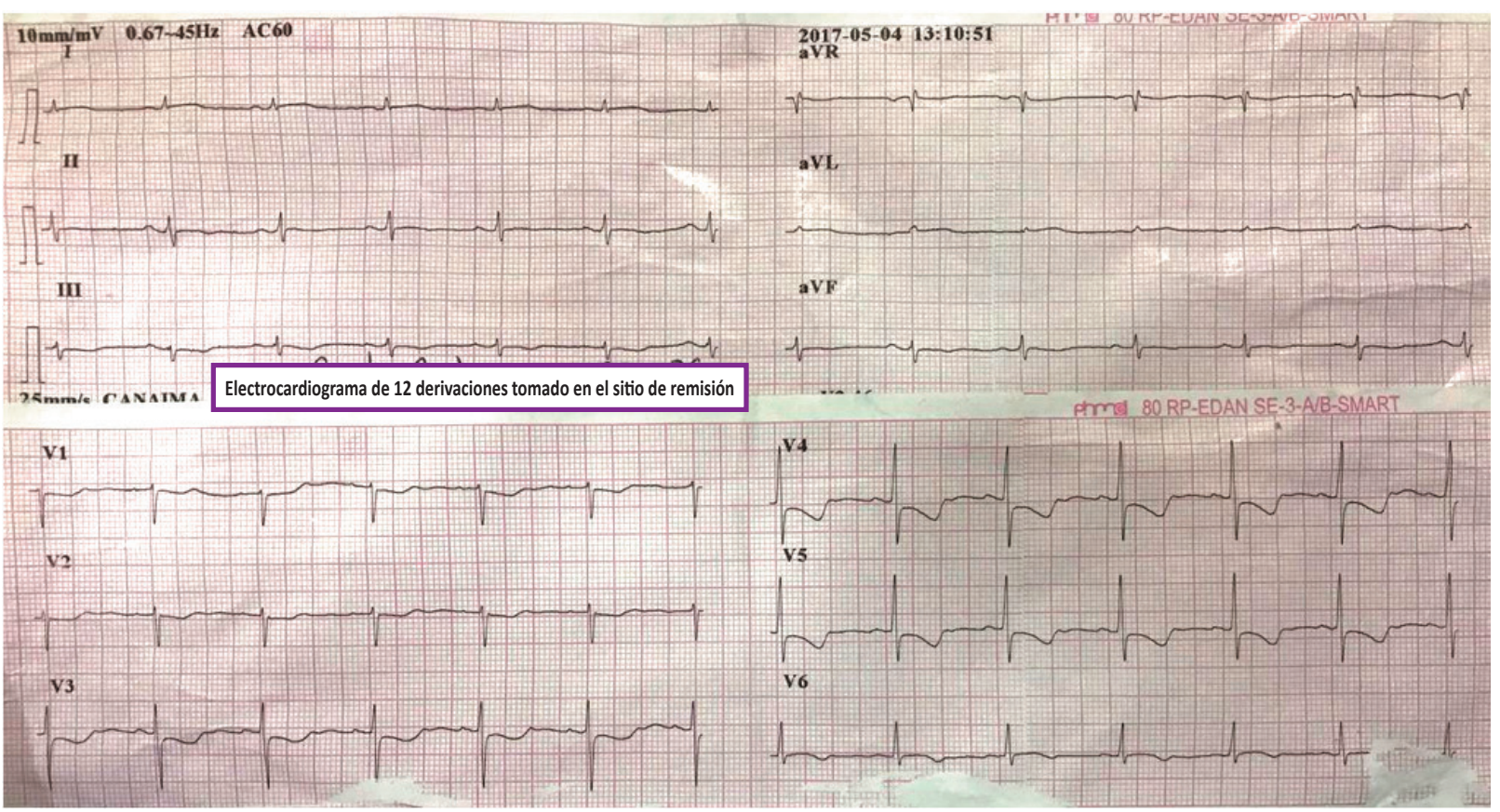

Figura 1. Electrocardiograma de 12 derivaciones tomado del sitio de remisión que muestra infra-desnivel de la cara antero lateral con una inversión asimétrica de la onda T; no se encontraron otras alteraciones significativas.

\section{INTRODUCCIÓN}

Las descripciones de los signos y síntomas cardiovasculares que se asocian a las reacciones alérgicas, anafilácticas y anafilactoides comenzaron a describirse en la literatura médica australiana, inglesa y alemana hace más de 70 años. Estas reacciones se debían a complicaciones asociadas al uso de "suero" ("enfermedad del suero") y la aplicación de la toxina tetánica en busca de protección inmunitaria; fueron descritas como "reacciones cardiacas morfológicas", "carditis aguda" o "lesiones con características básicas de carditis reumática" [1,2].

El primer reporte de un infarto agudo de miocardio asociado con un cuadro de urticaria data del año 1950 en un hombre que en encontraba recibiendo penicilina ${ }^{[3]}$. Sin embargo, fue hasta 1991 en que se realizó una descripción detallada del "síndrome angina alérgico" como un espasmo coronario, una manifestación de angina microvascular o disfunción endotelial y su progresión a infarto cardiaco agudo secundario a una reacción alérgica ${ }^{[4]}$. Posteriormente, estas entidades se catalogaron en el subgrupo de lesiones oclusivas dinámicas de las arterias coronarias ${ }^{[5]}$.

El síndrome de Kounis se define como la concurrencia de síndromes coronarios agudos (espasmo coronario, infarto agudo de miocardio y trombosis del stent) y condiciones asociadas con la activación de plaquetas, mastocitos y células inflamatorias interrelacionadas (linfocitos T y macrófagos) en el escenario de noxas anafilactoides/anafilácticas, de hipersensibilidad 0 alérgicas.

Esta no es una enfermedad rara sino una entidad clínica que se diagnostica de manera infrecuente debido a la falta de conocimiento de esta asociación, lo que condiciona el subregistro. Actualmente no existen guías de práctica clínica que establezcan el tratamiento de elección.

En este reporte presentamos el caso de una mujer de 46 años con de síndrome coronario agudo inducido por la administración de hioscina/dipirona, usada para el manejo sintomático de un dolor abdominal.

\section{REPORTE DE CASO}

Paciente mujer de 46 años que acudió a establecimiento de primer nivel de atención por presentar un cuadro clínico de cuatro días de evolución, caracterizado por dolor abdominal episódico -de pocos minutos de duración-, de tipo cólico, localizado inicialmente en hipocondrio derecho y que luego comprometió todo el abdomen, que aparecía generalmente luego del consumo de alimentos, con resolución espontánea y sin factores concomitantes; asociado a distensión abdominal.

Respecto a los antecedentes patológicos, la paciente negó presencia de enfermedades o factores de riesgo cardiovascular de importancia (sin hipertensión, sin dislipidemia, sin diabetes ni obesidad, sin historia familiar de enfermedad ateroesclerótica establecida y $\sin$ historia de tabaquismo).

Al examen físico de ingreso al hospital local, se notaron constantes vitales estables (presión arterial [PA]: 100/60 mmHg, frecuencia cardíaca [FC]: 84 latidos por minuto, frecuencia respiratoria [FR]: 20 latidos por minuto y temperatura [T]: $36,5^{\circ} \mathrm{C}$ ); no se encontraron anormalidades en el examen cardiovascular, a nivel 
Tabla 1. Paraclínicos tomados durante la observación del paciente. Se muestran la variación en los niveles de troponina.

\begin{tabular}{lcc}
\multicolumn{1}{c}{ Parámetro } & 04/05/2017 & 06/05/2017 \\
\hline Hemograma & & \\
Leucocitos (4 800-10 800 por $\mathrm{mm}^{3}$ ) & 9040 & 6900 \\
Neutrófilos (\%) & 68,8 & 43,5 \\
Linfocitos (\%) & 27,2 & 49,5 \\
Basófilos (\%) & 0,6 & 0,04 \\
Eosinófilos (\%) & 0,5 & 1,8 \\
Monocitos (\%) & 2,9 & 4,5 \\
Hemoglobina (G/DL) & 11,4 & 11,7 \\
Hematocrito (\%) & 34,3 & 35,1 \\
VCM (FL) & 101,8 & 101,4 \\
Plaquetas (150 000-450 000por mm ${ }^{3}$ ) & 219 & 245 \\
& & \\
Nitrógeno ureico (mg/dL) & 12 & 15 \\
Creatinina (mg/dL) & 0,8 & 0,7 \\
Troponina (0,012-0,034 ng/ml) & $10,9 *$ & -- \\
\hline
\end{tabular}

* Su valor disminuyó a 1,83 ng/ml al día siguiente.

abdominal no hubo evidencia de masas, "megalias" ni signos de irritación peritoneal.

Luego de la evaluación antes descrita, se consideró la presencia un cólico de origen biliar, por lo que el manejo sintomático consistió en la administración intravenosa de $20 \mathrm{mg} / 1 \mathrm{~g}$ de butil-bromuro de hioscina/dipirona. A los 15 minutos de la aplicación del medicamento, la paciente indicó la presencia de dolor torácico en región precordial de inicio súbito, opresivo, de intensidad 8/10 en la escala subjetiva del dolor, no referido, que se asoció diaforesis e hipotensión. A la evaluación física se encontró una PA: $80 / 56 \mathrm{mmHg}, \mathrm{FC}: 115$ latidos por minuto, FR: 24 respiraciones por minuto y $\mathrm{T}: 36,5^{\circ} \mathrm{C}$, además, lesiones dérmicas tipo habones en todo el cuerpo a predominio en tórax y sibilancias en auscultación pulmonar.

Los resultados del electrocardiograma de 12 derivaciones mostraron la presencia de infradesnivel del segmento ST en cara anterolateral (Figura 1). Se inició el manejo con hidrocortisona, oxígeno y líquidos endovenosos, y se decidió referirlo a una institución de mayor nivel de complejidad.

A su ingreso a la institución (hospital privado de tercer nivel de atención) no se encontraron mayores alteraciones en el examen físico; el electrocardiograma realizado se encontró dentro de parámetros normales con un segmento ST sin alteraciones (Figura 2), los biomarcadores de injuria miocárdica fueron positivos (Tabla 1).

La paciente fue trasladada a unidad de cuidados intensivos para monitorización cardiovascular y hemodinámica. El ecocardiograma transtorácico mostró cavidades auriculares y ventriculares de tamaño normal, sin masas ni trombos en su interior; una adecuada contractilidad de las paredes del ventrículo izquierdo y derecho, fracción de eyección calculada del $60 \%$ y sin alteraciones valvulares. La arteriografía coronaria documentó arterias coronarias epicárdicas sanas (Figura 3). Se indicó manejo sintomático. La paciente egresó luego de varios días de observación sin ninguna complicación.

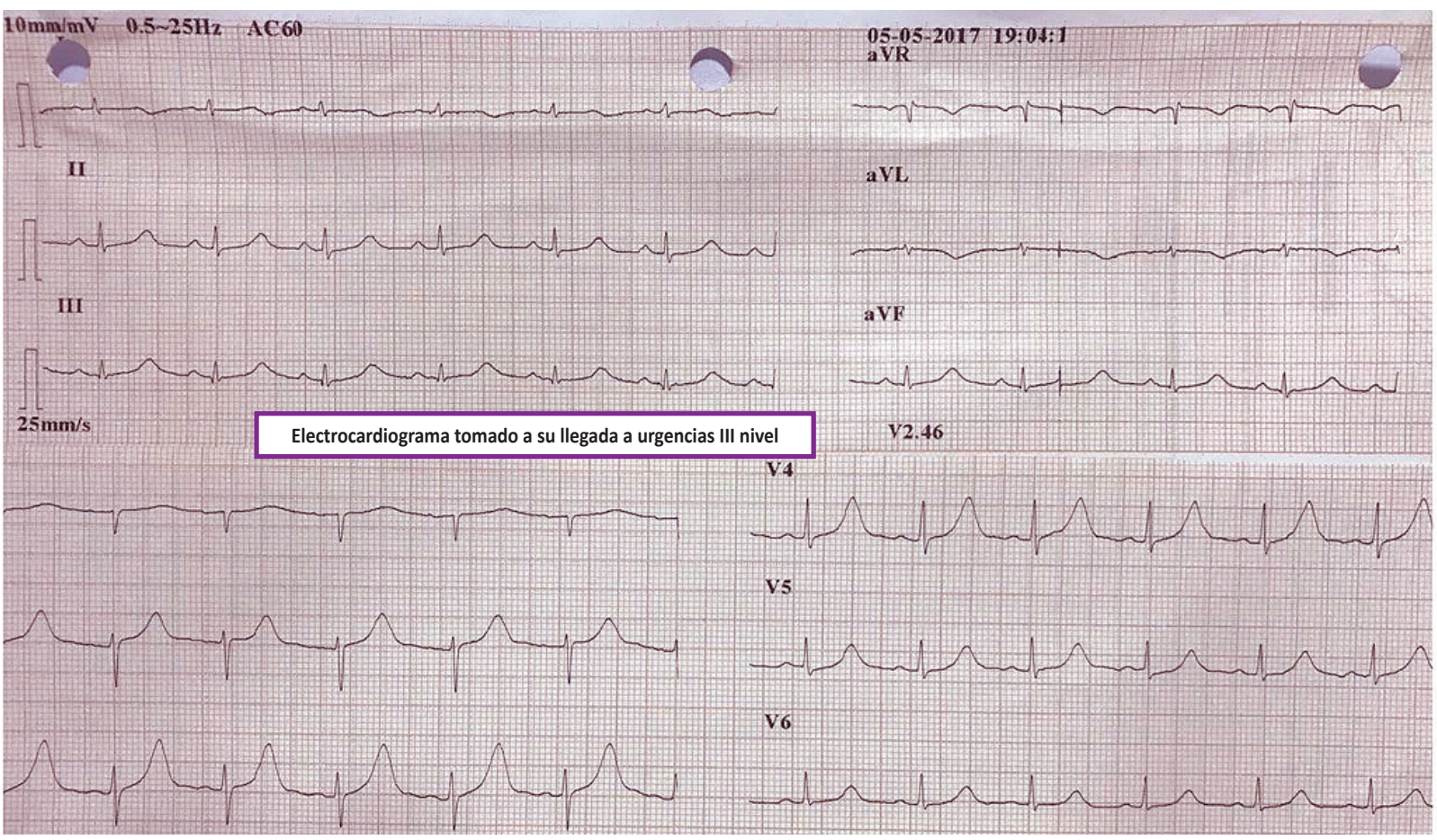

Figura 2. Electrocardiograma de control tomado a su llegada a la institución donde se evidencia que hay resolución de los cambios del segmento ST, no se encontraron ondas patológicas. 
Tabla 2. Hallazgos clínicos y de estudios reportados en síndrome de Kounis.

\begin{tabular}{ll}
\hline Síntomas & $\begin{array}{l}\text { Dolor torácico agudo, disconfort torácico, disnea, fatiga, cefalea, malestar general, nausea, } \\
\text { prurito, sincope, vomito }\end{array}$ \\
Tignos & Taquicardia, bradicardia, sudoración, diaforesis, hipotensión, frialdad de extremidades, \\
& palidez, palpitaciones, Rash dérmico. \\
& Fibrilación ventricular, fibrilación auricular, bloqueos de conducción cardiaca, bigeminismo, \\
taquicardia sinusal, bradicardia sinusal, elevación o depresión del segmento ST, \\
Cambios electrocardiográficos \\
aplanamiento o inversión de la onda T, prolongación del intervalo QT, ensanchamiento de \\
QRentriculares. \\
Angiografía coronaria: espasmo o trombosis \\
Eosinofilia en sangre periférica \\
Elevación de enzimas cardiacas: Creatin kinasa total y fracción MB \\
Elevación de troponinas \\
Cardiomegalia en radiografía de tórax \\
Cámaras cardiacas dilatadas en ecocardiograma \\
Eosinofilia y aumento de mastocitos en biopsias de especímenes coronarios \\
Resonancia nuclear magnética cardiaca: concentración subendocardica de gadolinio \\
PET (tomografía de emisión de positrones): defectos de isquemia.
\end{tabular}

Adaptado de Kounis NG ${ }^{[18]}$

\section{DISCUSIÓN}

Se describe el caso de una paciente femenina, sin factores de riesgo ni antecedentes de enfermedad cardiovascular, que presentó un evento coronario agudo asociado con una reacción anafiláctica secundaria a la aplicación de un medicamento. Esta asociación es conocida como síndrome de Kounis.

Numerosas causas han estado relacionadas con la aparición de esta enfermedad, entre las que se incluyen el consumo de ciertos medicamentos o alimentos, exposiciones medioambientales y ciertas condiciones de salud ${ }^{[6]}$. Dentro de estos tenemos: medicamentos (antibióticos, analgésicos especialmente los AINEs, antineoplásicos, medios de contraste, anestésicos endovenosos, trombolíticos, anticoagulantes, inhibidores de la bomba de protones), alimentos (pescado, frutas, vegetales, salsa de tomate), exposiciones ambientales (picaduras de hormigas, abejas, avispas, medusas, hiedra venenosa, veneno de víbora, metales, contacto con látex) y condiciones de salud (angioedema, asma, anafilaxia inducida por el ejercicio, el síndrome de ChurgStrauss).

Recientes reportes indican que esta enfermedad se presenta sin distinción de grupo étnico, etario (2 a 90 años) o localización geográfica. Por la falta de reconocimiento, no se cuentan con datos exactos que indiquen la incidencia y prevalencia de la enfermedad.

Un estudio prospectivo encontró que de 138911 pacientes admitidos durante un año, 793 presentaron cuadros alérgicos (769 por urticaria y 24 con angioedema); es decir, una incidencia de 5,7 por 1000 pacientes. De los 793 pacientes, 27 (3,4\%) correspondieron a casos que presentaron síndrome de Kounis, con una incidencia calculada de 19,4 casos por cada 10000 pacientes ${ }^{[7]}$.

Casi la mitad de los 51 casos de síndrome de Kounis que fueron reportados a la Agencia Internacional de Farmacovigilancia (VigiBase) durante el periodo de 2010-2014 ocurrieron en el último año, en su mayoría en Estados Unidos y estuvieron asociados al uso de antiinflamatorios no esteroideos ${ }^{[8]}$. Los datos de los laboratorios de hemodinamia reportaron incidencias muy bajas de la enfermedad $(0,002 \%)^{[9]}$.

La fisiopatología está relacionada con la desgranulación de mastocitos lo que genera la liberación de histamina, quimocinas, proteoglucanos, citoquinas, factores de crecimiento, serotonina, catepsina-D, triptasa, quimasa, leucotrienos, tromboxano, prostaciclina y factor activador plaquetario; todos estos agentes vasoactivos tienen múltiples efectos cardiovasculares ${ }^{[10-12]}$ que convergen para generar vasoespasmo coronario, disfunción endotelial y angina microvascular; además son capaces de erosionar una placa ateromatosa preexistente ${ }^{[9,13]}$.

De esta enfermedad se conocen tres categorías: el tipo I (sin enfermedad coronaria previa): incluye pacientes con arterias coronarias normales o casi normales sin factores predisponentes de enfermedad arterial coronaria, en quienes el ataque alérgico agudo puede inducir un espasmo coronario sin elevación de troponinas o un infarto agudo de miocardio ${ }^{[14]}$.

El tipo II (con enfermedad coronaria previa): aquellos pacientes con enfermedad ateromatosa preexistentes en quienes el ataque alérgico puede inducir a un espasmo coronario o la erosión o ruptura de la placa ateromatosa, manifestándose como un síndrome coronario agudo ${ }^{[14]}$. El tipo III, descrito 
recientemente, incluye a pacientes con trombosis de stent, en los que las muestras de tejido (trombo) en lámina, con tinciones de hematoxilina-eosina y de Giemsa, revelan la presencia de eosinófilos y mastocitos ${ }^{[15-17]}$.

Una variedad extensa de cambios del electrocardiograma que van desde elevación o depresión del segmento ST hasta bloqueos cardiacos de cualquier grado han sido reportados en la literatura ${ }^{[18,19]}$. En la Tabla 2 se muestran los principales hallazgos clínicos y paraclínicos de la enfermedad.

Para realizar el diagnostico se debe considerar el antecedente de reacciones alérgicas, una adecuada evaluación clínica y el apoyo exámenes auxiliares. A las pruebas usadas en la actualidad (electrocardiograma, ecocardiograma y la angiografía coronaria), se ha agregado la resonancia nuclear cardiaca. En los estudios paraclínicos, se pueden realizar pruebas de determinación de enzimas/mediadores de la respuesta de las células como mastocitos y eosinófilos, con la limitación de la cinética de la liberación y su uso no extenso ${ }^{[17,20]}$.

La medición de las enzimas cardiacas sugestivas de daño miocárdico es primordial como en los otros escenarios de síndrome coronario agudo; de estas la troponina es de elección por su especificidad y fácil accesibilidad. Algunos estudios sugieren su uso rutinario en todos los pacientes que acudan a emergencias por cuadros alérgicos con el fin de identificar de manera temprana a aquellos pacientes que presentan injuria o un evento coronario agudo ${ }^{[21,22]}$; lo anterior con el objetivo de realizar intervenciones oportunas y evitar el subdiagnóstico.

El ecocardiograma y la angiografía coronaria son necesarias para la identificación de anormalidades de la contractilidad y valorar la anatomía coronaria. Debe tenerse siempre presente, que otras patologías cardiacas relacionadas al estrés (síndrome de Takotsubo) pueden coexistir con el síndrome de Kounis; un estudio sugiere que podrían corresponder a una misma entidad ${ }^{[23]}$.
El manejo del síndrome de Kounis es un reto debido a la necesidad de tratamiento simultáneo de las manifestaciones alérgicas y cardiovasculares, debido a que fármacos con blanco a nivel cardiaco pueden empeorar los síntomas alérgicos y viceversa ${ }^{[24]}$. En pacientes con la variante tipo I, el tratamiento del evento alérgico puede aliviar los demás síntomas. El uso de corticoides (hidrocortisona) y de antihistamínicos $\mathrm{H} 1$ y $\mathrm{H} 2$ (difenhidramina y ranitidina) se puede considerar. Los vasodilatadores (bloqueadores de canales de calcio y nitratos) pueden controlar el vasoespasmo generado por la respuesta de hipersensibilidad. Se debe tener cuidado con el uso de la nitroglicerina que al inducir hipotensión y taquicardia puede empeorar el cuadro de anafilaxia ${ }^{[24]}$.

En los pacientes con síndrome de Kounis tipo II el tratamiento debe incluir el protocolo de síndrome coronario agudo junto con corticoides y antihistamínicos. Los beta-bloqueadores pueden exacerbar el espasmo coronario por su acción alfa adrenérgica. La epinefrina que es de elección en el escenario de la anafilaxia puede agravar la isquemia y empeorar el espasmo coronario. En casos severos se podría utilizar epinefrina libre de sulfato y en ruta intramuscular. Los pacientes con historia de cardiopatía isquémica y enfermedad coronaria quienes reciben beta bloqueadores pueden no responder al uso de epinefrina; en ellos se podría considerar utilizar glucagón en infusión o metoxamina (agonista alfa). Debe tenerse mucha precaución con el uso de opiáceos (que generan desgranulación masiva de mastocitos) que pueden empeorar la respuesta alérgica y no usar el acetaminofén que genera reducción del gasto cardiaco ${ }^{[24]}$.

Los pacientes con la tercera variante deben ser tratados siguiendo un protocolo de síndrome coronario agudo junto con aspiración urgente del trombo que deberá enviarse para tinciones especiales. Si hay síntomas alérgicos debe considerarse las intervenciones ya mencionadas ${ }^{[24]}$. Si los síntomas persisten se podría considerar la extracción del stent; si bien esto no se ha realizado en casos de Síndrome de Kounis específicamente, hay bibliografía que la

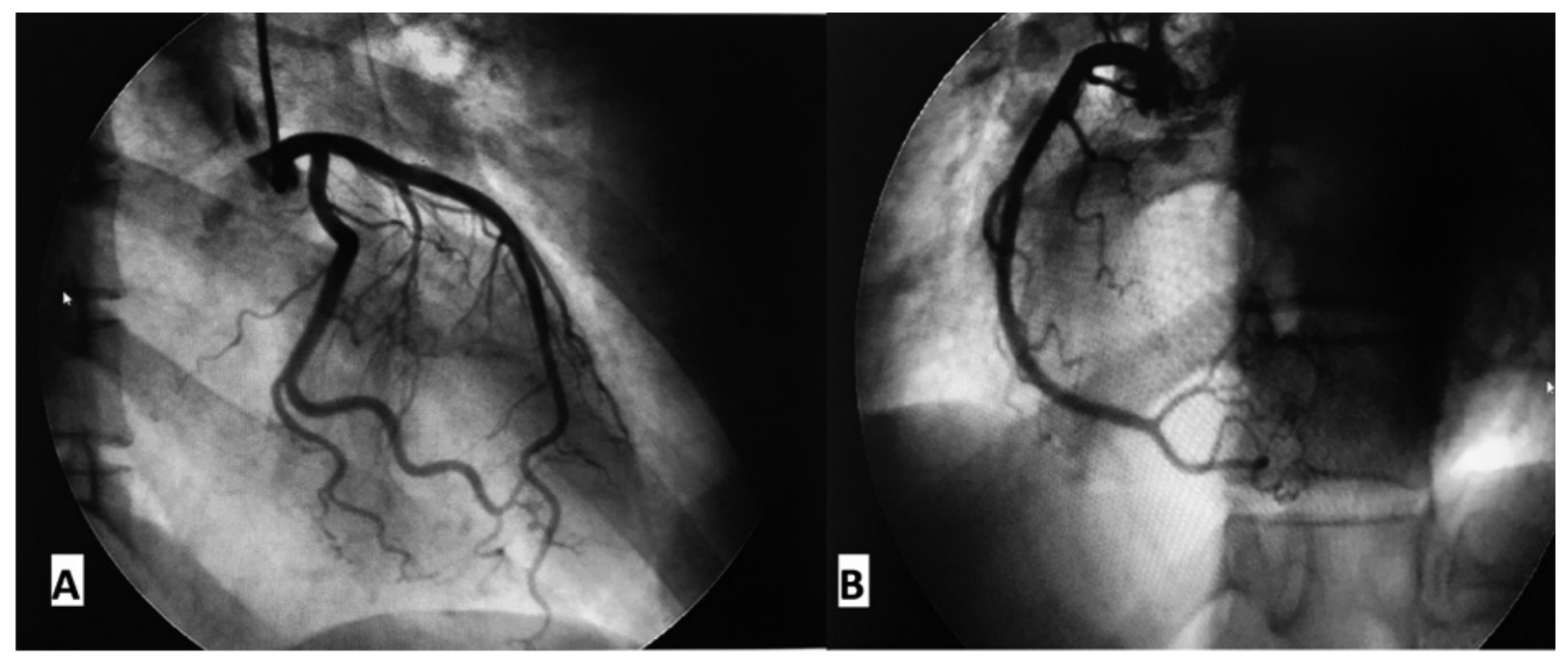

Figura 3. Angiografía coronaria. No se encontraron lesiones angiográficamente significativas. A) coronaria izquierda. B) coronaria derecha. 
respalda extrapolando la experiencia en el aumento de extracción de dichos dispositivos en pacientes con enfermedad coronaria e intervención coronaria percutánea previa y que luego requieren manejo quirúrgico de revascularización miocárdica abierta ${ }^{[25]}$.

Presentamos el caso de una paciente con síndrome de Kounis tipo $\mathrm{I}$, con un desencadenante farmacológico, sin factores de riesgo mayores de enfermedad coronaria, que luego de descartarse el evento coronario se vio una adecuada respuesta al manejo sintomático del cuadro alérgico.

Este síndrome es una condición médica que amenaza la vida de los pacientes y se encuentra subdiagnosticada por no considerarla dentro de los diagnósticos diferenciales. Se requiere de estudios futuros que permitan desarrollar guías de práctica clínica evitando complicaciones asociadas al uso erróneo de algunos medicamentos y establecer de forma exacta la prevalencia e incidencia a nivel mundial.

Conflicto de intereses. Los autores manifiestan no tener conflictos de interés.

\section{REFERENCIAS BIBLIOGRÁFICAS}

1. Schultheiss E. [Clinical aspects of allergic heart diseases]. Dtsch Med J. 1964;15:15-8. [Article in German]

2. Czickeli H. [Contribution to the problem of the allergic etiology of angina pectoris and myocardial infarct]. Klin Med Osterr Z Wiss Prakt Med. 1950;5(8):364-7.

3. Pfister $\mathrm{CW}$, Plice SG. Acute myocardial infarction during a prolonged allergic reaction to penicillin. Am Heart J. 1950;40(6):945-7.

4. Kounis NG, Zavras GM. Histamine-induced coronary artery spasm: the concept of allergic angina. Br J Clin Pract. 1991;45(2):121-8.

5. Braunwald E. Unstable angina: an etiologic approach to management. Circulation. 1998;98(21):2219-22.

6. Kounis NG, Mazarakis A, Tsigkas G, Giannopoulos S, Goudevenos $J$. Kounis syndrome: a new twist on an old disease. Future Cardiol. 2011;7(6):805-24.

7. Akoz A TH, Emet M, Bayramoglu A, Kizrak Y, Kantarci M, Aslan S. A prospective study of Kounis syndrome: clinical experience and cardiac magnetic resonance imaging findings for 21 patients. Acta Med Mediterr. 2013;29(4):811-6.

8. Renda F, Landoni G, Trotta F, Piras D, Finco G, Felicetti P, et al. Kounis Syndrome: An analysis of spontaneous reports from international pharmacovigilance database. Int J Cardiol. 2016;203:217-20.

9. Biteker M. Current understanding of Kounis syndrome. Expert Rev Clin Immunol. 2010;6(5):777-88.
10. Genovese A, Spadaro G. Highlights in cardiovascular effects of histamine and H1-receptor antagonists. Allergy. 1997;52(34 Suppl):67-78.

11. Theoharides TC, Kalogeromitros D. The critical role of mast cells in allergy and inflammation. Ann N Y Acad Sci. 2006;1088:78-99.

12. Steffel J, Akhmedov A, Greutert H, Luscher TF, Tanner FC. Histamine induces tissue factor expression: implications for acute coronary syndromes. Circulation. 2005;112(3):341-9.

13. Johnson JL, Jackson CL, Angelini GD, George SJ. Activation of matrix-degrading metalloproteinases by mast cell proteases in atherosclerotic plaques. Arterioscler Thromb Vasc Biol. 1998;18(11):1707-15.

14. Nikolaidis LA, Kounis NG, Gradman AH. Allergic angina and allergic myocardial infarction: a new twist on an old syndrome. Can J Cardiol. 2002;18(5):508-11.

15. Kounis NG, Kounis GN, Kouni SN, Soufras GD, Niarchos C, Mazarakis A. Allergic reactions following implantation of drug-eluting stents: a manifestation of Kounis syndrome? J Am Coll Cardiol. 2006;48(3):592-3.

16. Kounis NG, Hahalis G, Theoharides TC. Coronary stents, hypersensitivity reactions, and the Kounis syndrome. J Interv Cardiol. 2007;20(5):314-23.

17. Kounis NG, Almpanis G, Mazarakis A. Stent thrombosis and Kounis syndrome: who is guilty? J Cardiovasc Med (Hagerstown). 2011;12(1):71-2.

18. Kounis NG. Kounis syndrome: an update on epidemiology, pathogenesis, diagnosis and therapeutic management. Clin Chem Lab Med. 2016;54(10):1545-59.

19. Abdelghany $M$, Subedi R, Shah $S$, Kozman $H$. Kounis syndrome: A review article on epidemiology, diagnostic findings, management and complications of allergic acute coronary syndrome. Int J Cardiol. 2017;232:1-4.

20. Kounis NG. Serum tryptase levels and Kounis syndrome. Int J Cardiol. 2007;114(3):407-8.

21. Lippi G, Buonocore R, Schirosa F, Cervellin G. Cardiac troponin I is increased in patients admitted to the emergency department with severe allergic reactions. A case-control study. Int J Cardiol. 2015;194:68-9.

22. Cha YS, Kim H, Bang MH, Kim OH, Kim HI, Cha K, et al. Evaluation of myocardial injury through serum troponin I and echocardiography in anaphylaxis. Am J Emerg Med. 2016;34(2):140-4.

23. Kotsiou OS, Xirogiannis KI, Gourgoulianis KI. Kounis Syndrome: Is it Really a Takotsubo-Like Syndrome? J Investig Allergol Clin Immunol. 2017;27(3):198-200.

24. Cevik C, Nugent K, Shome GP, Kounis NG. Treatment of Kounis syndrome. Int J Cardiol. 2010;143(3):223-6.

25. Atoui R, Mohammadi S, Shum-Tim D. Surgical extraction of occluded stents: when stenting becomes a problem. Interact Cardiovasc Thorac Surg. 2009;9(4):736-8. 